Pacific Journal of Mathematics

GLOBAL EXISTENCE AND UNIQUENESS RESULTS FOR
SINGULAR SOLUTIONS OF THE CAPILLARITY EQUATION 


\title{
GLOBAL EXISTENCE AND UNIQUENESS RESULTS FOR SINGULAR SOLUTIONS OF THE CAPILLARITY EQUATION
}

\author{
MARIE-Francoise BidaUt-Veron
}

We study the singular solutions of the capillarity equation

$$
\operatorname{div} \frac{D v}{\sqrt{1+|D v|^{2}}}=K v \quad \text { in } \mathbf{R}^{N}
$$

with a $K<0$. We prove the global existence of a rotationally symmetric solution. We prove the uniqueness of a symmetric solution negative and concave near the origin.

Introduction. In this paper we study the existence and uniqueness of a singular solution of the capillarity equation in $\mathbf{R}^{N}$ :

$$
\operatorname{div}\left(D v /\left(\sqrt{1+|D v|^{2}}\right)=K v,\right.
$$

with a $K<0$. The situation is quite different from the case $K \geq 0$, where every isolated singularity is removable [4]. We restrict our attention to the symmetric case where $v$ depends only on the distance $r$ from the origin.

Let

$$
u(r)=\sqrt{-\frac{K}{N-1}} v\left(\sqrt{-\frac{N-1}{K}} r\right)
$$

Then the equation is equivalent to

$$
\left(\frac{r^{N-1} u^{\prime}}{\sqrt{1+u^{\prime 2}}}\right)^{\prime}(r)=-(N-1) r^{N-1} u(r) \text {. }
$$

In [1], P. Concus and R. Finn conjectured the global existence and uniqueness of a singular solution of (2). They proved the local existence of a function $u$ of the form

$$
u(r)=-\frac{1}{r}+\frac{N+3}{2(N-1)} r^{3}+r^{3} \varepsilon(r),
$$

where $\varepsilon(r)=o(r)$ when $r$ goes to 0 . Up to the change of $u$ into $-u$, they got local uniqueness in a particular class: functions such that $\varepsilon(r) / r^{p}$ $(p<4)$ and $r \varepsilon^{\prime}(r)$ are bounded. The solution has an asymptotic development in powers of $r$ but the formal Taylor series is divergent. 
In $\S 1$ we write the equation in terms of $z(r)=u^{\prime}(r) / \sqrt{1+u^{\prime 2}(r)}$, which leads us to a second order nonlinear equation:

$$
\Delta z(r)=(N-1) z(r)\left(\frac{1}{r^{2}}-\frac{1}{\sqrt{1-z^{2}(r)}}\right),
$$

with limit conditions $\lim _{r \rightarrow 0} z(r)=1, \lim _{r \rightarrow 0} z^{\prime}(r)=0$.

We give an a priori energy estimate for $z$ and $u$ in $\$ 2$.

Then, in $\$ 3$ we improve the results of local existence and uniqueness: we try to draw the maximum profit from the fixed point method introduced in [1], adapted to the function $z$. We get the local existence and uniqueness of functions $z$ such that $\left(z(r)-1+\left(r^{4} / 2\right)\right) / r^{6}$ is not too large, and then of functions $u$ such that $\left(u^{\prime}(r)-1 / r^{2}\right)$ is not too large. This result is an essential tool for uniqueness results of $\$ 5$.

In $\S 4$, from the energy estimate for $z$, we get global existence in $[0,+\infty$ [ for $z$, then for $u$. We study the behavior of $u, z$ for large $r$. They are oscillatory and go to zero when $r$ goes to infinity.

In $\S 5$, we prove the uniqueness of a solution $z$ nonincreasing near 0 , then the uniqueness of a solution $u$ concave near 0 . As the maximum principle fails, we use local comparison methods to obtain some accurate estimates near the origin, and prove that such functions $z, u$ are in the classes of uniqueness defined in $§ 3$.

1. New formulation of the problem. Up to the change of $u$ into $-u$, we shall deal with the existence and uniqueness of a singular symmetric solution of (2), negative near the origin. Let us recall the estimates given in [2]: every singular solution $u$ satisfies near the origin

$$
\begin{gathered}
-\left(\frac{\pi+\sqrt{2}}{\sqrt{N-1}} r+o(r)\right) \leq u(r)+\frac{1}{r} \leq \frac{\sqrt{2}}{\sqrt{N-1}} r+o(r), \\
\frac{u^{\prime}(r)}{\sqrt{1+u^{\prime 2}(r)}} \geq 1-\left(\frac{(\pi+\sqrt{2})^{2}}{2} r^{4}+o\left(r^{4}\right)\right) .
\end{gathered}
$$

Now we make a change of unknown function.

Proposition 1. The existence and uniqueness of a $C^{2}$ function $u$, singular symmetric solution of (1), is equivalent to the existence and uniqueness of a $C^{2}$ function $z$ solution of the second order semilinear elliptic equation:

$$
z^{\prime \prime}(r)+(N-1) \frac{z^{\prime}(r)}{r}=(N-1)\left(\frac{z(r)}{r^{2}}-\frac{z(r)}{\sqrt{1-z^{2}(r)}}\right),
$$


with limit conditions

$$
\lim _{r \rightarrow 0} z(r)=1 ; \quad \lim _{r \rightarrow 0} z^{\prime}(r)=0 .
$$

Functions $u$ and $z$ are linked by the relations

$$
\begin{gathered}
z(r)=\frac{u^{\prime}(r)}{\sqrt{1+u^{\prime 2}(r)}}=\sin \psi(r), \\
z^{\prime}(r)+(N-1) \frac{z(r)}{r}=-(N-1) u(r),
\end{gathered}
$$

where $\psi$ is the angle between the tangent at $(r, u(r))$ and the $r$ axis.

Proof. Let $u$ be a singular solution of (2) and $z$ be defined by (9). Then equation (2) takes the form (10), also equivalent to

$$
z(r)=-\frac{N-1}{r^{N-1}} \int_{0}^{r} \rho^{N-1} u(\rho) d \rho,
$$

since, from (5), (6), $r^{N-1} u(r)=O(1), r^{N-1} z(r)=o(1)$, when $r$ goes to 0 . Now (9) is obviously equivalent to

$$
u^{\prime}(r)=z(r) / \sqrt{1-z^{2}(r)}
$$

then we derive (10) and get (7); then (8) using (5), (6). Conversely let $z$ be a solution of (7), (8) and define $u$ by (10); then $u$ satisfies (12), (9), then (2), and $u(r) \sim{ }_{r \rightarrow 0}-1 / r$, so that $u$ is singular.

2. A priori estimates. Now we get an estimate of the energy for $z$, which later on will be fundamental.

Proposition 2. Let $z$ be a solution of (7), (8), defined on an interval $[0, R[$. Then

$$
g(r)=\frac{z^{\prime 2}(r)}{2(N-1)}+\frac{1-z^{2}(r)}{2 r^{2}}-\sqrt{1-z^{2}(r)}<0,
$$

and $g^{\prime}(r)<0$ in ]0, $R$ [. Consequently

$$
\begin{gathered}
o<\sqrt{1-z^{2}(r)}<2 r^{2}, \\
\left.\left|z^{\prime}(r)\right|<\sqrt{N-1} \min (r, \sqrt{2}), \text { in }\right] 0, R[.
\end{gathered}
$$

Proof. Multiplying (7) by $z^{\prime}(r)$, we get

$$
g^{\prime}(r)=-\frac{z^{\prime 2}(r)}{r}-\frac{1-z^{2}(r)}{r^{3}}<0,
$$


since $z^{2}(r)<1$; multiplying (7) by $r^{2} z^{\prime}(r)$, we get also

$$
\left(r^{2} g\right)^{\prime}(r)=-\left(\frac{N-2}{N-1} z^{\prime 2}(r)+2 r \sqrt{1-z^{2}(r)}\right)<0
$$

now from (8) we have $\lim _{r \rightarrow 0} r^{2} g(r)=0$, then $r^{2} g(r)<0$ in $] 0, R[$; hence (13) and (14). Then (15) follows from the fact that

$$
2 g(r)=\frac{z^{\prime 2}(r)}{N-1}+\left(\frac{\sqrt{1-z^{2}(r)}}{r}-r\right)^{2}-r^{2} \text {. }
$$

Consequences.

(a) We obtain other estimates for $z$ and $u$ in $] 0, R[$ :

$$
1>z(r)>1-\frac{\sqrt{N-1}}{2} r^{2},
$$

from (8), (15), and

$$
-\frac{r}{\sqrt{N-1}}<u(r)+\frac{1}{r}<\frac{N+1}{2 \sqrt{N-1}} r
$$

from (10), (19).

Now from (14), (19) and (20), we deduce

$$
\begin{gathered}
r^{2} \leq \max \left(\frac{1}{2}, \frac{2}{\sqrt{N-1}}\right) \Rightarrow z(r)>0 \Rightarrow u^{\prime}(r)>0, \\
r^{2}<2 \frac{\sqrt{N-1}}{N+1} \Rightarrow u(r)<0 \Rightarrow z(r)>0 \Rightarrow u^{\prime}(r)>0 .
\end{gathered}
$$

(b) We can improve the local estimates (5), (6): from (10), (14) and (15) we get, near the origin,

$$
\begin{gathered}
1>z(r)>1-\left(2 r^{4}+o\left(r^{4}\right)\right), \\
-\frac{r}{\sqrt{N-1}}<u(r)+\frac{1}{r}<\frac{r}{\sqrt{N-1}}+O\left(r^{3}\right) .
\end{gathered}
$$

REMARK. Let us note an estimate of the energy for $u$, which has often been used in [2], [3]: let

$$
f(r)=\frac{u^{2}(r)}{2}-\frac{\sqrt{1-z^{2}(r)}}{N-1}
$$

then

$$
\left.f^{\prime}(r)=-\frac{z^{2}(r)}{r \sqrt{1-z^{2}(r)}}<0 \text { in }\right] 0, R[
$$


hence for any $r, s \in] 0,+\infty[$ such that $r>s$,

$$
\frac{u^{2}(r)}{2}-\frac{\sqrt{1-z^{2}(r)}}{N-1} \leq \frac{u^{2}(s)}{2}-\frac{\sqrt{1-z^{2}(s)}}{N-1} \text {. }
$$

3. Local existence and uniqueness. From Proposition 1, and (3), (9), we still obtain the local existence of a solution $Z$ of the problem (7), (8) of the form

$$
Z(r)=1-r^{4} / 2+O\left(r^{8}\right)
$$

near the origin. Now we prove a quite more accurate result, based on a fixed point method analogous to [1].

TheOREM 1. Let $M<M_{0}=(N+8) / 3 \sqrt{N-1}$. Then, for $R_{0}$ sufficiently small, the problem (7), (8) admits a unique $C^{2}$ solution $Z$ in $\left.] 0, R_{0}\right]$ such that

$$
\left\{\begin{array}{l}
Z(r)=1-r^{4} / 2+r^{6} w(r) \\
\left.|w(r)| \leq M \quad \text { in }] 0, R_{0}\right]
\end{array}\right.
$$

Proof. Let for any $y \in]-1,+1[$ and $r>0$

$$
\Phi(y, r)=(N-1)\left(\frac{y}{r^{2}}-\frac{y}{\sqrt{1-y^{2}}}\right) \text {. }
$$

Let $M<M_{0}, R>0$, and denote

$$
B_{M, R}=\left\{v \in C^{0}([0, R])\left|\|v\|=\max _{r \in[0, R]}\right| v(r) \mid \leq M\right\} .
$$

Then one can see as in [1] that the problem is equivalent to a fixed point problem: find a function $w \in B_{M, R}$ such that

$$
w=T(w),
$$

where

$$
\begin{aligned}
& T(w)(r) \\
& =\frac{r^{-(N+8) / 2}}{\sqrt{N-1}} \int_{0}^{r} \tau^{(N+2) / 2} F(w(\tau), \tau) \sin \frac{\sqrt{N-1}}{2}\left(\frac{1}{\tau^{2}}-\frac{1}{r^{2}}\right) d \tau \\
& F(w, r)=2(N+2) r^{2}+\frac{N(N-4)}{4} r^{4} w+(N-1) w \\
& +\Phi\left(1-\frac{r^{4}}{2}+r^{6} w, r\right) .
\end{aligned}
$$


Let $w \in B_{M, R}$. Then there exists $\theta(r) \in[0,1]$ such that

$$
\begin{aligned}
\Phi\left(1-\frac{r^{4}}{2}+r^{6} w(r), r\right)= & \Phi\left(1-\frac{r^{4}}{2}, r\right)+r^{6} w(r) \frac{\partial \Phi}{\partial y}\left(1-\frac{r^{4}}{2}, r\right) \\
& +r^{12} \frac{w^{2}(r)}{2} \frac{\partial^{2} \Phi}{\partial y^{2}}\left(1-\frac{r^{4}}{2}+r^{6} \theta(r) w(r), r\right) .
\end{aligned}
$$

Now

$$
\begin{aligned}
& \frac{\partial \Phi}{\partial y}(y, r)=(N-1)\left(\frac{1}{r^{2}}-\left(1-y^{2}\right)^{-3 / 2}\right), \\
& \frac{\partial^{2} \Phi}{\partial y^{2}}(y, r)=-3(N-1) y\left(1-y^{2}\right)^{-5 / 2},
\end{aligned}
$$

hence for sufficiently small $r$,

$$
\begin{aligned}
\Phi\left(-1+\frac{r^{4}}{2}+r^{6} w(r), r\right) & \\
=(N-1)\left(-\frac{r^{2}}{8}+\right. & O\left(r^{6}\right) \\
+ & \left.\frac{5}{8} r^{4} w(r)-w(r)-\frac{3}{2} r^{2} w^{2}(r)\left(1+O\left(r^{2}\right)\right)\right), \\
r^{(N+2) / 2} F(w(r), r)= & \frac{15 N+33}{8} r^{(N+6) / 2} \\
& +\frac{(N+1)(2 N-5)}{8} r^{(N+10) / 2} w(r) \\
& -\frac{3(N-1)}{2} r^{(N+6) / 2} w^{2}(r)\left(1+O\left(r^{2}\right)\right) .
\end{aligned}
$$

Then we integrate by parts the first term, cf. [2], and get

$$
T(w)(r)=\frac{15 N+33}{8(N-1)} r^{2}+O\left(r^{4}\right)+O\left(r^{2}\right)+R(r)=O\left(r^{2}\right)+R(r),
$$

with

$$
|R(r)| \leq \frac{r^{-(N+8) / 2}}{\sqrt{N-1}} \frac{3(N-1)}{2}\|w\|^{2} \int_{0}^{r} \tau^{(N+6) / 2} d \tau=\frac{\|w\|^{2}}{M_{0}} \leq \frac{M}{M_{0}} .
$$

As $M<M_{0}$, we deduce that there exists $R_{1}=R_{1}(M)>0$ such that $T$ maps $B_{M, R}$ into itself for $R \leq R_{1}$. 
Moreover, let $w, \hat{w} \in B_{M, R_{1}}$; then there exists $\eta, \xi \in B_{M, R_{1}}$ such that $\eta(r) \in[w(r), \hat{w}(r)]$ and

$$
\begin{aligned}
& \Phi\left(1-\frac{r^{4}}{2}+r^{6} \hat{w}(r), r\right)-\Phi\left(1-\frac{r^{4}}{2}+r^{6} w(r), r\right) \\
& =r^{6}(\hat{w}(r)-w(r)) \frac{\partial \Phi}{\partial y}\left(1-\frac{r^{4}}{2}+r^{6} \eta(r), r\right) \\
& =(\hat{w}(r)-w(r))\left(r^{6} \frac{\partial \Phi}{\partial y}\left(1-\frac{r^{4}}{2}, r\right)+r^{12} \eta(r) \frac{\partial^{2} \Phi}{\partial y^{2}}\left(1-\frac{r^{4}}{2}+r^{6} \xi(r), r\right)\right) \\
& =(N-1)\left(-1+\frac{5}{8} r^{4}+O\left(r^{6}\right)-3 r^{2} \eta(r)\left(1+O\left(r^{2}\right)\right)\right)(\hat{w}(r)-w(r)) \\
& =(N-1)\left(-1-3 r^{2} \eta(r)+O\left(r^{4}\right)\right)(\hat{w}(r)-w(r)),
\end{aligned}
$$

hence

$$
\begin{aligned}
& r^{(N+2) / 2}(F(\hat{w}(r), r)-F(w(r), r)) \\
& =\left(-3(N-1) r^{(N+6) / 2} \eta(r)+O\left(r^{(N+10) / 2}\right)\right)(\hat{w}(r)-w(r)), \\
& |T(\hat{w})(r)-T(w)(r)| \leq\left(\frac{2}{M_{0}} \max (\|w\|,\|\hat{w}\|)+O\left(r^{2}\right)\right)\|\hat{w}-w\| .
\end{aligned}
$$

Then for any $\varepsilon>0$ there exists $R_{0}=R_{0}(\varepsilon, M)<R_{1}$ such that if $R \leq R_{0}$,

$$
\|T(\hat{w})-T(w)\| \leq\left(\frac{2}{M_{0}} \max (\|w\|,\|\hat{w}\|)+\varepsilon\right)\|\hat{w}-w\|
$$

and

$$
\|T(w)\| \leq \varepsilon M_{0}+\frac{\|w\|^{2}}{M_{0}} .
$$

Then $\left\|T^{n}(w)\right\| \leq v_{n}$ where $v_{n}=\varepsilon M_{0}+\left(v_{n-1}^{2} / M_{0}\right), \quad v_{0}=M$. Now take $\varepsilon<\min \left(\left(M / M_{0}^{2}\right)\left(M_{0}-M\right), 1 / 6\right) ;$ then $v_{n} \searrow \lambda$ where $\lambda=$ $\left(M_{0} / 2\right)(1-\sqrt{1-4 \varepsilon})<2 \varepsilon M_{0}<M_{0} / 3$. Then

$$
\left\|T^{n}(\hat{w})-T^{n}(w)\right\| \leq a_{n}\|\hat{w}-w\|,
$$

where

$$
a_{n}=\prod_{k=0}^{n}\left(\frac{2 v_{n}}{M_{0}}+\varepsilon\right) ; \quad \lim _{n \rightarrow+\infty} \frac{a_{n+1}}{a_{n}}=\frac{2 \lambda}{M_{0}}+\varepsilon<1,
$$

then $\lim _{n \rightarrow+\infty} a_{n}=0$; hence for large $n, T^{n}$ is a strict contraction. Then $T$ has a unique fixed point in $B_{M, R_{0}}$. 
REMARK. As in [1], we can prove that the function $Z$ has an asymptotic development near 0 in powers of $r^{4}$ whose first terms are

$$
Z(r)=1-\frac{r^{4}}{2}+\frac{15 N+33}{8(N-1)} r^{8}+o\left(r^{8}\right)
$$

Now from (7)

$$
\left(r^{N-1} Z^{\prime}\right)^{\prime}(r)=r^{N-1} \Phi(Z(r), r),
$$

hence $Z^{\prime}$, then $Z^{\prime \prime}$ and all the derivatives of $Z$ have an asymptotic development near 0 , obtained by successive differentiations of the development of $Z$, and $Z$ is in $C^{\infty}\left(\left[0, R_{0}\right]\right)$. Indeed, by recursion the derivatives cannot have a development with negative powers of $r$. Then with equation (7) we obtain by recursion all the terms of the development and deduce the divergence of the Taylor series. Now observe that

$$
Z^{\prime}(r)=-2 r^{3}+o\left(r^{3}\right), \quad Z^{\prime \prime}(r)=-6 r^{2}+o\left(r^{3}\right),
$$

so that $Z^{\prime}(r)$ and $Z^{\prime \prime}(r)$ are negative near the origin.

Theorem 1 is still an improvement of the results in [1]. Let us apply it to the function $u$.

Corollary 1. Let $\tilde{M}<M_{0}$. Then for $\tilde{R}_{0}$ sufficiently small, the problem (2) admits a unique $C^{2}$ solution $U$ in $\left.10, \tilde{R}_{0}\right]$, singular, such that

$$
\left\{\begin{array}{l}
U^{\prime}(r)=\frac{1}{r^{2}}+\omega(r), \\
\left.|\omega(r)| \leq \tilde{M} \quad \text { in }] 0, \tilde{R}_{0}\right] .
\end{array}\right.
$$

Proof. Let $\tilde{M}<M_{0}$ and $M<\tilde{M}$, and $U$ be the singular solution of (2) associated with the solution $Z$ defined by (28). Then by calculation

$$
U^{\prime}(r)=\frac{Z(r)}{\sqrt{1-Z^{2}(r)}}=\frac{1}{r^{2}}+w(r)+O\left(r^{2}\right),
$$

hence for $\tilde{R}_{0}$ sufficiently small $U$ satisfies (34). Let $u$ be another singular solution satisfying (34) in $\left.] 0, \tilde{R}_{0}\right]$ and $z$ be the solution of (7) (8) associated to $u$. Then by calculation

$$
z(r)=\frac{u^{\prime}(r)}{\sqrt{1+u^{\prime 2}(r)}}=1-\frac{r^{4}}{2}+r^{6} \omega(r)+o\left(r^{6}\right) .
$$


Then for $R$ sufficiently small

$$
\left\{\begin{array}{l}
z(r)=1-\frac{r^{4}}{2}+r^{6} w(r), \\
\left.\left.|w(r)| \leq \frac{\tilde{M}+M_{0}}{2} \text { in }\right] 0, R\right]
\end{array}\right.
$$

hence $z(r)=Z(r)$ near the origin, hence in $\left.] 0, \tilde{R}_{0}\right]$.

4. Global existence and asymptotic properties. Here we prove the existence of global solutions.

THEOREM 2. Each solution $z$ of (7)(8), or equivalently each singular solution $u$ of (2), admits a unique extension defined on the whole interval ]0, $+\infty[$.

Proof. From Proposition 1 we have only to consider $z$. Let $z$ be a solution of (7) (8) defined on an interval $[0, R)$. Let $x=\left(z, z^{\prime}\right)$, then equation (7) takes the form

$$
x^{\prime}(r)=G(r, x(r)),
$$

where $G$ is a $C^{1}$ function on the open set $\left.W=\right] 0,+\infty[\times]-1,+1[\times \mathbb{R}$. Then $z$ admits a unique maximal extension, still called $z$, defined on an interval $\left[0, R_{m}\right)$.

Suppose $R_{m}<+\infty$. From (15), $z^{\prime}$ is bounded; hence $z(r)$ has a limit $z_{m}$ when $r \nearrow R_{m}$. From Proposition 2, the energy function $g$, decreasing and bounded below by -1 , has a limit $\gamma<0$. By contradiction this implies $z_{m} \neq \pm 1$. Then, from (7), $z^{\prime \prime}$ is bounded near $R_{m}$, hence $z^{\prime}(r)$ has a limit $z_{m}^{\prime}$. Then $\left(R_{m}, z_{m}, z_{m}^{\prime}\right) \in W$, hence $z$ admits an extension to an interval $\left[0, R_{m}+\varepsilon\right)$, which is impossible.

Now we make precise the behavior near infinity of any solution:

THEOREM 3. Each solution $z$ of (7), (8) admits a countable number of zeros, asymptotically separated by a distance of $\pi / \sqrt{N-1}$, and

$$
\begin{gathered}
\frac{z^{2}(r)+z^{\prime 2}(r)}{r} \in L^{1}(] a,+\infty[), \quad \text { for any } a>0, \\
\lim _{r \rightarrow+\infty} z(r)=\lim _{r \rightarrow+\infty} z^{\prime}(r)=\lim _{r \rightarrow+\infty} u(r)=\lim _{r \rightarrow+\infty} u^{\prime}(r)=0, \\
\frac{u^{2}(r)+u^{\prime 2}(r)}{r} \in L^{1}(] a,+\infty[), \quad \text { for any } a>0 .
\end{gathered}
$$


Proof. Let $z$ be a solution of (7), (8) on [0, $+\infty$ [. From Proposition 2, the energy function $g$ has a limit $\gamma<0$ when $r$ goes to $+\infty$. By contradiction, this implies that $\liminf _{r \rightarrow+\infty} \sqrt{1-z^{2}(r)}>0$. Then there exists $\alpha>0$ such that $\sqrt{1-z^{2}(r)}>\alpha$ for large $r$.

Let us make the substition $z=r^{-(N-1) / 2} y$ in equation (7); this equation becomes

$$
y^{\prime \prime}(r)+p(r) y(r)=0,
$$

where

$$
p(r)=(N-1)\left(\frac{1}{\sqrt{1-z^{2}(r)}}-\frac{N+1}{4 r^{2}}\right) ;
$$

for large $r$, we have $(N-1) / 2<p(r)<(N-1) / \alpha$; hence, from the Sturm comparison theorem, $z$ is oscillatory; moreover, let

$$
0<r_{1}<r_{2}<\cdots<r_{n}<r_{n+1}<\cdots
$$

be the zeros of $z$, simple because of the local uniqueness in (35); then the distance $d_{n}=r_{n+1}-r_{n}$ between two consecutive zeros satisfies

$$
\sqrt{\alpha} \frac{\pi}{\sqrt{N-1}}<d_{n}<\sqrt{2} \frac{\pi}{\sqrt{N-1}} .
$$

Moreover for any $r_{n}$ such that $r_{n} \geq 1-$ if $N=2$ there is no condition since $r_{1}>1$ from $(21)$ - there exists a unique point $\left.s \in\right] r_{n}, r_{n+1}$ [ where $z^{\prime}\left(s_{n}\right)=0$ : indeed, if not, there would exist an $\left.r \in\right] r_{n}, r_{n+1}[$ such that

$$
0=\left(r^{N-1} z^{\prime}\right)^{\prime}(r)=r^{N-1} z(r)\left(\frac{1}{r^{2}}-\frac{1}{\sqrt{1-z^{2}(r)}}\right),
$$

and then $z(r)=0$, which is impossible.

On the other hand, from (16) we deduce that, for any $a>0, z^{\prime 2}(r) / r$ $\in L^{1}(] a,+\infty[)$. In the same way, the function $f$ defined in (25) is decreasing and bounded below by $-1 /(N-1)$; hence it has a limit when $r$ goes to $+\infty$; then from (26) we deduce that

$$
\frac{z^{2}(r)}{r \sqrt{1-z^{2}(r)}} \in L^{1}(] a,+\infty[),
$$

hence (36).

Now let us prove (37), (38). Suppose first that $\gamma=-1$; then

$$
\lim _{r \rightarrow+\infty}\left(\frac{z^{\prime 2}(r)}{2\left(N^{r}-1\right)}+\frac{1-z^{2}(r)}{2 r^{2}}+1-\sqrt{1-z^{2}(r)}\right)=0,
$$


then $\lim _{r \rightarrow+\infty} z^{\prime}(r)=\lim _{r \rightarrow+\infty} z(r)=0$. From (11) and (13) we get (37) and (38).

Suppose now that $\gamma>-1$; we will obtain a contradiction. For the extremal points $s_{n}$ of $z$ on $\left[r_{n}, r_{n+1}\right]$ we have $\lim _{n \rightarrow+\infty} \sqrt{1-z\left(s_{n}\right)^{2}}=-\gamma$ $\in] 0,1\left[\right.$, then $\left.\lim _{n \rightarrow+\infty}\left|z\left(s_{n}\right)\right|=k \in\right] 0,1\left[\right.$. Let $\sigma_{n}$ be the unique point of ]$r_{n}, s_{n}\left[\right.$ where $z\left(\sigma_{n}\right)=z\left(s_{n}\right) / 2$. Then, from (15),

$$
\left|\frac{z\left(s_{n}\right)}{2}\right|=\left|z\left(\sigma_{n}\right)-z\left(r_{n}\right)\right| \leq \sqrt{2(N-1)}\left(\sigma_{n}-r_{n}\right) .
$$

Hence with (41) we get for large $n$

$$
\frac{k}{4 \sqrt{N-1}}<\sigma_{n}-r_{n}<\frac{\sqrt{2 \pi}}{\sqrt{N-1}} .
$$

Now for any $r \in\left[r_{n}, \sigma_{n}\right], \sqrt{1-z^{2}(r)} \geq \sqrt{1-z^{2}\left(s_{n}\right) / 4}$, then from the expression of $g$,

$$
\frac{z^{\prime 2}(r)}{2(N-1)} \geq g(r)-\frac{1-z^{2}(r)}{2 r^{2}}+\sqrt{1-\frac{z^{2}\left(s_{n}\right)}{4}} ;
$$

let $\mu=\gamma+\sqrt{\gamma^{2}+3} / 2>0$; hence for large $n$

$$
z^{\prime 2}(r) \geq 2(N-1) \mu .
$$

From (42), (43) we deduce that for $n_{0}$ sufficiently large,

$$
\int_{n_{0}}^{+\infty} \frac{z^{\prime 2}(r)}{r} d r \geq \sum_{n \geq n_{0}} \int_{r_{n}}^{\sigma_{n}} \frac{z^{\prime 2}(r)}{r} d r \geq \frac{\sqrt{N-1}}{2} k \mu \sum_{n \geq n_{0}} \frac{1}{\sigma_{n}} .
$$

Now from (41), (42), $\sigma_{n}=O\left(r_{n}\right)=O(n)$. This is impossible, since $z^{\prime 2}(r) / r$ is integrable on $] n_{0},+\infty[$.

Finally, we have $\lim _{r \rightarrow+\infty} p(r)=(N-1)$, since $\lim _{r \rightarrow+\infty} z(r)=0$. From the Sturm comparison theorem we get

$$
\lim _{n \rightarrow+\infty}\left(d_{n}-\frac{\pi}{\sqrt{N-1}}\right)=0
$$

REMARKS.

(i) Obviously the function $u$ admits a countable number of zeros $\rho_{n}$, such that, from (22):

$$
0<\rho_{1}<r_{1}<\rho_{2}<r_{2}<\cdots<\rho_{n}<r_{n}<\rho_{n+1}<r_{n+1} \cdots ;
$$

on $\left[\rho_{n}, \rho_{n+1}\right], u$ has a unique extremum in $r_{n}$. From (27) we get $\left|u\left(r_{n}\right)\right|>$ $\left|u\left(r_{n+1}\right)\right|$, that is to say $\left|z^{\prime}\left(r_{n}\right)\right|>\left|z^{\prime}\left(r_{n+1}\right)\right|$, for any $n$. 
Moreover $f\left(r_{1}\right)<f\left(\rho_{1}\right)$; this or (15) implies, cf. [5]:

$$
0<u\left(r_{1}\right)=-\frac{z^{\prime}\left(r_{1}\right)}{N-1}<\sqrt{2 /(N-1)} .
$$

(ii) Consider for simplification the case $N=2$. The function $p$ defined by (40) satisfies $p(r)>\left(1-\frac{3}{4} r^{2}\right)$. In the Bessel equation of order 1 ,

$$
\zeta^{\prime \prime}(r)+\frac{\zeta^{\prime}(r)}{r}=\frac{\zeta(r)}{r^{2}}-\zeta(r),
$$

we make the substition $\zeta=r^{-1 / 2} \xi$; this equation becomes

$$
\xi^{\prime \prime}(r)+\left(1-\frac{3}{4 r^{2}}\right) \xi(r)=0 .
$$

From the Sturm comparison theorem, between two successive zeros in ] $0,+\infty$ [ of any Bessel function of order 1, there exists at least one zero of $z$; in fact exactly one for large $r$ since the zeros of the Bessel functions are asymptotically separated by $\pi$. Likewise between 0 and the first zero $R_{1} \neq 0$ of the function $J_{1}$, there exists at least one zero of $z$ (if not, for any $\varepsilon \in] 0, R$, we would have, with $\xi=r^{1 / 2} J_{1}$,

$$
\left[y \xi^{\prime}-\xi y^{\prime}\right]_{\varepsilon}^{R_{1}}=\int_{\varepsilon}^{R_{1}}\left(p(r)-1+\frac{3}{4} r^{2}\right) y(r) \xi(r) d r>0 ;
$$

now $\xi(\varepsilon)=O\left(\varepsilon^{3 / 2}\right), y^{\prime}(\varepsilon)=O\left(\varepsilon^{-1 / 2}\right)$, hence $\lim _{\varepsilon \rightarrow 0} \xi(\varepsilon) y^{\prime}(\varepsilon)=0$;

$$
\lim _{\varepsilon \rightarrow 0} y(\varepsilon) \xi^{\prime}(\varepsilon)=\lim _{\varepsilon \rightarrow 0} z(\varepsilon) \varepsilon^{1 / 2}\left(\varepsilon^{1 / 2} J_{1}^{\prime}(\varepsilon)+\varepsilon^{-1 / 2} \frac{J_{1}(\varepsilon)}{2}\right)=0,
$$

hence $y\left(R_{1}\right) \xi^{\prime}\left(R_{1}\right)>0$, which is impossible since $\left.y\left(R_{1}\right)>0, \xi^{\prime}\left(R_{1}\right)<0\right)$.

Using (22), we deduce the estimates

$$
\sqrt{2 / 3}<\rho_{1}<r_{1}<R_{1} \simeq 3.8 ; \quad \sqrt{2}<r_{1} ;
$$

notice that for the solutions $Z$ and $U$ we get numerically $\rho_{1} \cong 1.5$, $r_{1} \cong 2.8$.

It is an open question whether the extremal points of the function $z$ satisfy $\left(z\left(s_{n}\right)\right)=O\left(1 / \sqrt{s_{n}}\right)$, as is the case for Bessel functions.

5. Uniqueness under growth conditions. We have seen in $\$ 4$ that the solution $Z$ defined in Theorem 1 is a decreasing function for small $r$. Differentiating (12), we get

$$
u^{\prime \prime}(r)=z^{\prime}(r) /\left(1-z^{2}(r)\right)^{3 / 2},
$$


so that the solution $U$ is strictly concave for small $r$. We are going to prove reciprocally that any solution $z$ nonincreasing for small $r$ is equal to $Z$, any solution $u$ concave for small $r$ is equal to $U$ :

THEOREM 4. There is a unique solution $z$ of (7)(8) in $] 0,+\infty[$ such that $z$ is nonincreasing near the origin. There is a unique singular solution $u$ of (2) in $] 0,+\infty[$ such that $u$ is concave near the origin.

Proof. Step 1. An estimate for $z$.

Let $z$ be a solution such that $z^{\prime}(r) \leq 0$ in an interval $] 0, \alpha$ [; in terms of $u$, that means from (49) that $u^{\prime \prime}(r) \leq 0$ in ]0, $\alpha$. Let $\left.\rho \in\right] 0, \alpha$ [be fixed. We are going to compare $z$ to a function $\bar{w}$ of the form

$$
\bar{w}(r)=a r^{2}+b r+c r^{1-N},
$$

such that

$$
\bar{w}(\rho)=z(\rho), \quad \bar{w}^{\prime}(\rho)=z^{\prime}(\rho), \quad \bar{w}^{\prime \prime}(\rho)=z^{\prime \prime}(\rho) .
$$

We find

$$
\left\{\begin{array}{l}
a=-\frac{N-1}{N+1} \frac{z(\rho)}{\sqrt{1-z^{2}(\rho)}}, \\
b=\frac{1}{N}\left((N-1) \frac{z(\rho)}{\rho}+z^{\prime}(\rho)+(N-1) \frac{\rho z(\rho)}{\sqrt{1-z^{2}(\rho)}}\right) \\
c=\frac{\rho^{N-1}}{N}\left(z(\rho)-\rho z^{\prime}(\rho)-\frac{N-1}{N+1} \rho^{2} \frac{z(\rho)}{\sqrt{1-z^{2}(\rho)}}\right)
\end{array}\right.
$$

Then from equation (7) we get

$$
\begin{aligned}
\left((\bar{w}-z)^{\prime}\right. & \left.+\frac{N-1}{r}(\bar{w}-z)\right)^{\prime}(r) \\
= & (N-1)\left(\frac{z(r)}{\sqrt{1-z^{2}(r)}}-\frac{z(\rho)}{\sqrt{1-z^{2}(\rho)}}\right) \\
= & (N-1)\left(u^{\prime}(r)-u^{\prime}(\rho)\right) .
\end{aligned}
$$

As $u^{\prime}$ is nonincreasing, we deduce from (51) that

$$
\begin{aligned}
& (\bar{w}-z)^{\prime}(r)+\frac{N-1}{r}(\bar{w}-z)(r) \\
& \left.\quad=r^{1-N}\left(r^{N-1}(\bar{w}-z)\right)^{\prime}(r) \leq 0, \text { in }\right] 0, \alpha[,
\end{aligned}
$$


and then that

$$
(\bar{w}-z)(r)(r-\rho) \leq 0, \text { in }] 0, \alpha[.
$$

As $z$ is nonincreasing we deduce that

$$
(\bar{w}(r)-z(\rho))(r-\rho) \leq 0, \text { in }] 0, \alpha[.
$$

Let $k=r / \rho$. Then

$$
(k-1)(\bar{w}(k \rho)-z(\rho)) \leq 0 \text { in }] 0, \alpha / \rho[.
$$

From (50), (52), we obtain

$$
\begin{array}{r}
\bar{w}(k \rho)-z(\rho) \\
=\frac{k^{1-N}}{N}\left[z(\rho)\left((N-1) k^{N}-N k^{N-1}+1\right)+\rho z^{\prime}(\rho)\left(k^{N}-1\right)\right. \\
\left.\quad-\frac{N-1}{N+1} \frac{\rho^{2} z(\rho)}{\sqrt{1-z^{2}(\rho)}}\left(N k^{N+1}-(N+1) k^{N}+1\right)\right] \\
=\frac{k^{1-N}}{N}(k-1)^{2}\left(z(\rho) P(k)+\frac{\rho z^{\prime}(\rho)}{k-1} Q(k)-\frac{\rho^{2} z(\rho)}{\sqrt{1-z^{2}(\rho)}} R(k)\right),
\end{array}
$$

where

$$
\left\{\begin{array}{l}
P(k)=(N-1) k^{N-2}+(N-2) k^{N-3}+\cdots+2 k+1, \\
Q(k)=k^{N-1}+k^{N-2}+\cdots+1 \\
R(k)=\frac{N-1}{N+1}\left(N k^{N-1}+(N-1) k^{N-2}+\cdots+2 k+1\right) .
\end{array}\right.
$$

As $z$ is positive near 0 , we obtain the inequalities, for sufficiently small $\rho$,

$$
\begin{cases}\frac{\rho^{2}}{\sqrt{1-z^{2}(\rho)}} R(k) \geq P(k)+\frac{\rho z^{\prime}(\rho)}{z(\rho)} \frac{Q(k)}{k-1}, & \text { if } k \in] 1, \alpha / \rho[, \\ \frac{\rho^{2}}{\sqrt{1-z^{2}(\rho)}} R(k) \leq P(k)+\rho \frac{z^{\prime}(\rho)}{z(\rho)} \frac{Q(k)}{k-1}, & \text { if } k \in] 0,1[.\end{cases}
$$

Take first $k=1+\rho$, for sufficiently small $\rho$. From the majorization (16) we obtain

$$
\begin{aligned}
& \frac{\rho^{2}}{\sqrt{1-z^{2}(\rho)}} \frac{N(N-1)}{2}\left(1+\frac{2(N-1)}{3} \rho+o(\rho)\right) \\
& \quad \geq \frac{N(N-1)}{2}\left(1+\frac{2(N-2)}{3} \rho+o(\rho)\right)-N \sqrt{N-1}(\rho+o(\rho)),
\end{aligned}
$$


hence we get the estimate

$$
\sqrt{1-z^{2}(\rho)} \leq \rho^{2}+2\left(\frac{1}{3}+\frac{1}{\sqrt{N-1}}\right) \rho^{3}+o\left(\rho^{3}\right) .
$$

Now take $k=1-\rho$. Then we get in the same way the estimate

$$
\sqrt{1-z^{2}(\rho)} \geq \rho^{2}-2\left(\frac{1}{3}+\frac{1}{\sqrt{N-1}}\right) \rho^{3}+o\left(\rho^{3}\right) .
$$

Hence

$$
\sqrt{1-z^{2}(\rho)}=\rho^{2}+O\left(\rho^{3}\right)
$$

so we still sharpen the estimate (14).

Step 2. Improvement of the estimates.

Consider first a point $\rho$ where $z^{\prime}(\rho) \geq-C\left(\rho^{3}+o\left(\rho^{3}\right)\right)$ for a $C>0$. Take $k=1+q \rho^{2}$, where $q$ is a parameter. Then from (56) we get

$$
\begin{aligned}
& \frac{\rho^{2}}{\sqrt{1-z^{2}(\rho)}} \frac{N(N-1)}{2}\left(1+\frac{2(N-1)}{3} q \rho^{2}+o\left(\rho^{2}\right)\right) \\
& \geq \frac{N(N-1)}{2}\left(1+\frac{2(N-2)}{3} q \rho^{2}+o\left(\rho^{2}\right)\right)-N \frac{C}{q}\left(\rho^{2}+o\left(\rho^{2}\right)\right),
\end{aligned}
$$

hence, taking $q=\sqrt{3 C /(N-1)}$ for the better estimate, we get

$$
\sqrt{1-z^{2}(\rho)} \leq \rho^{2}+4 \sqrt{\frac{3 C}{N-1}} \rho^{4}+o\left(\rho^{4}\right),
$$

and, in the same way, with $k=1-q \rho^{2}$,

$$
\sqrt{1-z^{2}(\rho)} \geq \rho^{2}-4 \sqrt{\frac{3 C}{N-1}} \rho^{4}+o\left(\rho^{4}\right) .
$$

Now consider the function $\varphi=\psi^{2}$, where,

$$
\psi(r)=\frac{r^{2}-\sqrt{1-z^{2}(r)}}{r^{4}}
$$

then

$$
\begin{aligned}
& \varphi^{\prime}(r)=2 \psi(r) \psi^{\prime}(r) \\
& \quad=2 \psi(r) \frac{r^{-5}}{\sqrt{1-z^{2}(r)}}\left(r z(r) z^{\prime}(r)-2 r^{2} \sqrt{1-z^{2}(r)}+4\left(1-z^{2}(r)\right)\right) .
\end{aligned}
$$

Observe that there exists no neighborhood of 0 where $\psi(r) \leq 0$ : suppose $\psi(r) \leq 0$ in $] 0, \beta]$; from (7) we have

$$
r^{1-N}\left(r^{N-1} z^{\prime}\right)^{\prime}(r)=(N-1) z(r) \frac{\sqrt{1-z^{2}(r)}-r^{2}}{r^{2} \sqrt{1-z^{2}(r)}},
$$


hence, from (8), $r^{N-1} z^{\prime}$ would be nondecreasing near 0 , then $z$ would be nondecreasing near 0 ; hence $z(r)=1, \psi(r)=r^{-2}$ near 0 , which is impossible.

Now consider three cases:

First case. There exists $\alpha>0$ such that $\left.\left.\varphi^{\prime}(r) \neq 0, \forall r \in\right] 0, \alpha\right]$. Then $\psi(r) \neq 0$, hence $\psi(r)>0, \forall r \in] 0, \alpha]$. Moreover we have $\varphi^{\prime}(r)>0, \forall r$ $\in] 0, \alpha]$ : if not, we would have $\varphi(r)>\varphi(\alpha)>0$, hence $r^{2}-\sqrt{1-z^{2}(r)}$ $>\psi(\alpha) r^{4}$, then from (8), (59) and (63)

$$
\left(r^{N-1} z^{\prime}\right)^{\prime}(r)<-\frac{N-1}{2} \psi(\alpha) r^{N-1},
$$

near the origin; and integrating twice

$$
z(r) \leq 1-\frac{N-1}{4 N} \psi(\alpha) r^{2},
$$

which is in contradiction with (23).

Now take $\rho$ sufficiently small; since $\psi^{\prime}(\rho)>0$, we have

$$
\begin{aligned}
z^{\prime}(\rho) & >\frac{1}{z(\rho)}\left(2 \rho \sqrt{1-z^{2}(\rho)}-4 \frac{1-z^{2}(\rho)}{\rho}\right) \\
& \geq-2 \rho^{3}(1+O(\rho))
\end{aligned}
$$

then from (60) (61) we get the estimate

$$
\left|\sqrt{1-z^{2}(\rho)}-\rho^{2}\right| \leq 4 \sqrt{\frac{2}{3(N-1)}} \rho^{4}+o\left(\rho^{4}\right) .
$$

Second case. For any $\alpha>0$ there exists $r<\alpha$ such that $\psi(r)=0$. Then there exists $r_{1}<1$ such that $\psi\left(r_{1}\right)=0$. There exists $r_{2}<r_{1}$ such that $\psi\left(r_{2}\right)>0$. Consider a small $\rho<r_{2}$; then there exists $r_{3}<\rho$ such that $\psi\left(r_{3}\right)=0$. The function $\varphi$ has a maximum on $\left[r_{3}, r_{1}\right]$ in a point $\bar{\rho}$ such that $\varphi(\bar{\rho})>\varphi\left(r_{2}\right)>0$. Then $\psi^{\prime}(\bar{\rho})=0$, hence

$$
z^{\prime}(\bar{\rho})=-2 \bar{\rho}^{3}(1+O(\bar{\rho})),
$$

so that we have the estimate (64) at point $\bar{\rho}$, that is to say

$$
|\psi(\bar{\rho})| \leq 4 \sqrt{\frac{2}{3(N-1)}}+o(1)
$$

then $|\psi(\rho)| \leq \psi(\bar{\rho})$, hence we get the estimate (64) at the point $\rho$.

Third case. There exists $\alpha_{0}>0$ such that $\psi(r)>0$ in ]0, $\alpha_{0}$ ], and for any $\alpha>0$, there exists $r<\alpha$ such that $\varphi^{\prime}(r)=0$. Then there exists 
$r_{1}<\alpha_{0}$ such that $\varphi^{\prime}\left(r_{1}\right)=0$. Consider a small $\rho<r_{1}$; there exists $r_{2}<\rho$ such that $\varphi^{\prime}\left(r_{2}\right)=0$. The function $\varphi$ has a maximum in $\left[r_{2}, r_{1}\right]$ in a point $\bar{\rho}$ such that $\varphi^{\prime}(\bar{\rho})=0$, hence $\psi^{\prime}(\bar{\rho})=0$. Hence we have again (64) at $\bar{\rho}$, then at $\rho$.

Step 3. Conclusion.

Consequently in any case we have the estimate (64). We deduce easily that, near the origin:

$$
z(\rho)=1-\frac{\rho^{4}}{2}+\rho^{6} w(\rho)
$$

with

$$
|w(\rho)| \leq 4 \sqrt{\frac{2}{3(N-1)}}+o(1) .
$$

Now let us remember that the constant which defines the class of uniqueness in $\S 3$ is $M_{0}=(N+8) / 3 \sqrt{N-1}$, and observe that $4 \sqrt{(2 / 3(N-1))}<M_{0}$ for any $N \geq 2$. Then from Theorem 1 , we deduce that $z$ is equal to $Z$, hence $u$ is equal to $U$, near the origin, and on the whole interval $] 0,+\infty[$.

\section{REFERENCES}

[1] P. Concus and R. Finn, A singular solution of the capillarity equation, I: Existence, Invent. Math., 29 (1975), 143-148.

[2] A singular solution of the capillarity equation, II: Uniqueness, Invent. Math., 29 (1975), 149-160.

[3] _ The shape of a pendent liquid drop, Philos. Trans. Roy. Soc. London A, 292 (1979), 307-340.

[4] R. Finn, On partial differential equations whose solutions admit no isolated singularities, Scripta Math., 26 (1961), 107-115.

[5] _ Some properties of capillarity free surfaces, Seminar on Minimal Submanifolds, Princeton Univ. Press (1983), 323-337.

[6] _ On the pendent liquid drop, preprint (1984).

[7] E. Giusti, The pendent water drop. A direct approach, Boll. Un. Mat. Ital., 17A (1980), 458-465.

Received July 2, 1985.

UNIVERSITE DE TOURS

PARC DE GRANDMONT

37200 TOURS, France 



\title{
PACIFIC JOURNAL OF MATHEMATICS \\ EDITORS
}

\author{
V. S. VARADARAJAN \\ (Managing Editor) \\ University of California \\ Los Angeles, CA 90024 \\ HERBERT CLEMENS \\ University of Utah \\ Salt Lake City, UT 84112 \\ R. FINN \\ Stanford University \\ Stanford, CA 94305
}

\author{
HERMANN FLASCHKA \\ University of Arizona \\ Tucson, AZ 85721
}

RAMESh A. GANGOLLI

University of Washington Seattle, WA 98195

VAUGHAN F. R. JONES

University of California

Berkeley, CA 94720

ROBION KIRBY

University of California

Berkeley, CA 94720
C. C. MOORE

University of California

Berkeley, CA 94720

H. SAMELSON

Stanford University

Stanford, CA 94305

HAROLD STARK

University of California, San Diego

La Jolla, CA 92093

\section{ASSOCIATE EDITORS}

\author{
R. ARENS \\ E. F. BECKENBACH \\ B. H. NeUmanN \\ F. WOLF \\ K. YOSHIDA \\ (1906-1982)

\section{SUPPORTING INSTITUTIONS} \\ UNIVERSITY OF ARIZONA \\ UNIVERSITY OF BRITISH COLUMBIA \\ CALIFORNIA INSTITUTE OF TECHNOLOGY \\ UNIVERSITY OF CALIFORNIA \\ MONTANA STATE UNIVERSITY \\ UNIVERSITY OF NEVADA, RENO \\ NEW MEXICO STATE UNIVERSITY \\ OREGON STATE UNIVERSITY \\ UNIVERSITY OF OREGON \\ UNIVERSITY OF SOUTHERN CALIFORNIA \\ STANFORD UNIVERSITY \\ UNIVERSITY OF HAWAII \\ UNIVERSITY OF TOKYO \\ UNIVERSITY OF UTAH \\ WASHINGTON STATE UNIVERSITY \\ UNIVERSITY OF WASHINGTON
}

The Supporting Institutions listed above contribute to the cost of publication of this Journal, but they are not owners or publishers and have no responsibility for its content or policies.

Mathematical papers intended for publication in the Pacific Journal of Mathematics should be in typed form or offset-reproduced (not dittoed), double spaced with large margins. Please do not use built up fractions in the text of the manuscript. However, you may use them in the displayed equations. Underline Greek letters in red, German in green, and script in blue. The first paragraph must be capable of being used separately as a synopsis of the entire paper. In particular it should contain no bibliographic references. Please propose a heading for the odd numbered pages of less than 35 characters. Manuscripts, in triplicate, may be sent to any one of the editors. Please classify according to the scheme of Math. Reviews, Index to Vol. 39. Supply name and address of author to whom proofs should be sent. All other communications should be addressed to the managing editor, or Elaine Barth, University of California, Los Angeles, California 90024.

There are page-charges associated with articles appearing in the Pacific Journal of Mathematics. These charges are expected to be paid by the author's University, Government Agency or Company. If the author or authors do not have access to such Institutional support these charges are waived. Single authors will receive 50 free reprints; joint authors will receive a total of 100 free reprints. Additional copies may be obtained at cost in multiples of 50 .

The Pacific Journal of Mathematics is issued monthly as of January 1966. Regular subscription rate: $\$ 190.00$ a year (5 Vols., 10 issues). Special rate: $\$ 95.00$ a year to individual members of supporting institutions.

Subscriptions, orders for numbers issued in the last three calendar years, and changes of address should be sent to Pacific Journal of Mathematics, P.O. Box 969, Carmel Valley, CA 93924, U.S.A. Old back numbers obtainable from Kraus Periodicals Co., Route 100, Millwood, NY 10546.

The Pacific Journal of Mathematics at P.O. Box 969, Carmel Valley, CA 93924 (ISSN 0030-8730) publish'es 5 volumes per year. Application to mail at Second-class postage rates is pending at Carmel Valley, California, and additional mailing offices. Postmaster: send address changes to Pacific Journal of Mathematics, P.O. Box 969, Carmel Valley, CA 93924.

PUBLISHED BY PACIFIC JOURNAL OF MATHEMATICS, A NON-PROFIT CORPORATION

Copyright (C) 1986 by Pacific Journal of Mathematics 


\section{Pacific Journal of Mathematics}

\section{Vol. 125, No. $2 \quad$ October, 1986}

Dale Edward Alspach, On $\mathscr{L}_{p, \lambda}$ spaces for small $\lambda \ldots \ldots \ldots \ldots \ldots \ldots 257$

Jong Sook Bae and Sangsuk Yie, Range of Gateaux differentiable operators

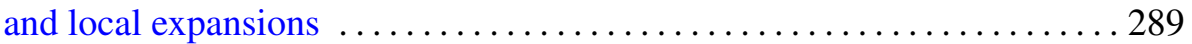

Hubert Berens and Lothar Hetzelt, On accretive operators on $l_{n}^{\infty} \ldots \ldots 301$

Marie-Françoise Bidaut-Véron, Global existence and uniqueness results for singular solutions of the capillarity equation ................. 317

Donald M. Davis and Mark Mahowald, Classification of the stable homotopy types of stunted real projective spaces ................. 335

Aad Dijksma, Heinz K. Langer and Hendrik S. V. de Snoo, Unitary

colligations in $\Pi_{\kappa}$-spaces, characteristic functions and Štraus extensions

Michel Enock and Jean-Marie Schwartz, Algèbres de Kac

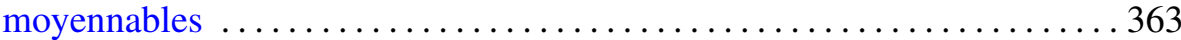

Seppo Granlund, Peter Lindqvist and Olli Martio, Note on the

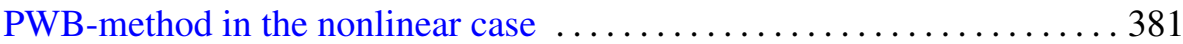

Palle E. T. Jorgensen, Analytic continuation of local representations of Lie

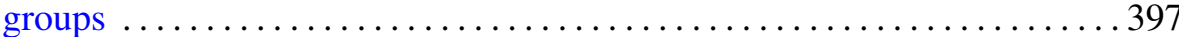

Robert P. Kaufman, Plane curves and removable sets ................409

José M. Montesinos and Wilbur Carrington Whitten, Constructions of two-fold branched covering spaces $\ldots \ldots \ldots \ldots \ldots$.

Benedict Seifert, Highly transitive group actions on trees and normalizing Tits systems

Charles Stuart Stanton, Counting functions and majorization for Jensen

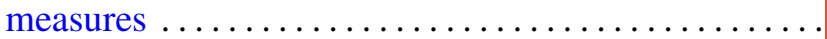

Luen-Fai Tam, On existence criteria for capillary free surfaces without gravity

Zhuocheng Yang, Exposed points of left invariant means 